\title{
Feature-Level Fusion of Hand Biometrics for Personal Verification Based on Kernel PCA
}

\author{
Qiang Li, Zhengding Qiu, and Dongmei Sun \\ Institute of Information Science, \\ Beijing Jiaotong University, Beijing 100044, P.R. China \\ liqianglqa126.com
}

\begin{abstract}
This paper presents a novel method of feature-level fusion (FLF) based on kernel principle component analyze (KPCA). The proposed method is applied to fusion of hand biometrics include palmprint, hand shape and knuckleprint, and we name the new feature as "handmetric". For different kind of samples, polynomial kernel is employed to generate the kernel matrixes that indicate the relationship among them. While fusing these kernel matrixes by fusion operators and extracting principle components, the handmetric feature space is established and nonlinear feature-level fusion projection could be implemented. The experimental results testify that the method is efficient for feature fusion, and could keep more identity information for verification.
\end{abstract}

\section{Introduction}

Fusion of different kind of data for a better decision is a hot topic in many research areas. While in the filed of personal authentication, multimodal biometric technology is becoming an important approach to alleviate the problems intrinsic to stand-alone biometric systems. According to Jain and Ross [1], the information of different biometrics could be fused in three levels: feature extraction level, matching score level and decision level. Though feature-level fusion (FLF) could keep the identity information to its most and expected to perform better than at the other two levels, the study on it is seldom reported. There are mainly two reasons of it [12]. First, the feature spaces of different biometric traits may not compatible. That is, different features may have different dimension and measurement, and their dynamic variation ranges lie in different complicated nonlinear spaces. Second, FLF may lead to the "curse of dimensionality" problem by concatenating several features as one. While solving these problems, we propose a new strategy for FLF based on KPCA.

The choice and number of biometric traits is another issue in multimodal biometric system. In this paper, fusion of hand based biometrics including palmprint [10][11], hand geometry and knuckleprint [3] are investigated. All these three biometrics have the advantage of robust to noise and change of environment, and available in lowresolution images $(<50 \mathrm{dpi})$. Furthermore, they could be extracted in the same original hand image and overcome several disadvantages of multimodal biometrics such as inconvenience to use, parameters drifting for different capture devices and higher database requirement. 


\section{Hand Feature Extraction and Fusion Algorithm}

\subsection{Handmetric: Palmprint, Hand Geometry and Knuckleprint}

Palmprint contains the majority of identity information in low-resolution hand images, and could achieve rather high accuracy [10][11]. As one of the most reliable biometric traits, palmprint and have got widely attention in recent years. However, palmprint recognition is still underdevelopment. Besides the problem of device size, illumination and environment variation, there are mainly four difficulties in palmprint recognition: a) ROI (region of interest) is hard to determine precisely; b) change of hand pose brings about variable shadow and nonlinear distortion, which is hard to eliminate; c) the feature lines/points are hard to define exactly; d) palmprint may change dynamically during long period of time.

Fuse with other kind of biometric, palmprint recognition could be more accurate and more ready to business use. By adding hand geometry information on palmprint, Kumar et al. [4] suggests that fusion in decision level using max rule could outperform traditional serial concatenate FLF method and single palmprint system. Ribaric et al. [5] testifies weighted sum rule in a similar manner. Based on these works, we define the outline of the hand and cuticle in the inner surface of the hand as "handmetric". As to low-resolution images, handmetric contains palmprint, hand geometry and knuckleprint with their geometrical correlativity. In this paper, handmetric refers to the FLF of three kind of hand biometrics. Because hand shape is mainly "finger shape", it can be combined with knuckleprint easily [3]. Without loss of generality, we choose the image of middle finger (as shown in Fig.1 (a)) to obtain the whole finger feature. While fusing palmprint (as shown in Fig.1 (b)) with finger feature by FLF, handmetric feature could be obtained for personal verification.

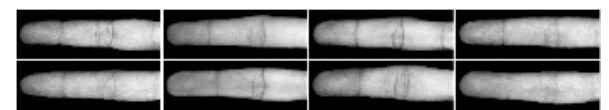

(a)

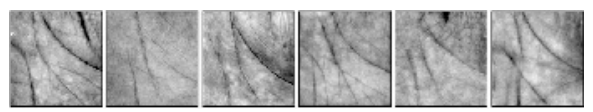

(b)

Fig. 1. Image examples in handmetric database: (a) the middle finger images from different persons, (b) the palmprint images from different persons

\subsection{KPCA Algorithm}

KPCA is the combination of kernel projection and PCA dimension reduction method [6]. It has been widely used in subspace feature extraction, especially in face recognition task [7]. The main idea of KPCA is: transform the original sample space $\mathbf{X}$ into high dimensional space $\mathbf{D}$ by applying a nonlinear mapping $\Phi$ to each of the samples. PCA is then performed in $\mathbf{D}$ to get a feature space $\mathbf{F}$. KPCA is the nonlinear extent of PCA, and PCA can be interpreted as a linear version of KPCA.

Given $M$ original training samples $x_{1}, x_{2}, \ldots, x_{M} \in R^{N}$, the space $\mathbf{D}$ should be $\left\{\Phi\left(x_{i}\right) \mid\right.$ $i=1, \ldots, M\}$. The correlation matrix of $\mathbf{D}$ is defined as kernel matrix $\mathbf{K}$ :

$$
K_{i j}=\left(\Phi\left(x_{i}\right) \cdot \Phi\left(x_{j}\right)\right) \quad i, j=1,2, \cdots, M
$$


K can be regarded as generalized similarity measure among the data [2]. Centering the embeddings in $\mathbf{D}$ is needed for further processing. According to [6], centering could acts on $\mathbf{K}$ and can be implemented by operator $\mathcal{D}$ :

$$
\tilde{K}_{i j}=\mathcal{D}(\mathbf{K})=\left[\mathbf{K}-\mathbf{1}_{M} \mathbf{K}-\mathbf{K} \mathbf{1}_{M}+\mathbf{1}_{M} \mathbf{K} \mathbf{1}_{M}\right]_{i j}
$$

where $\left[\mathbf{1}_{M}\right]_{i j}=1 / M$ for all $i, j$. Then eigenvalues and eigenvectors of $\widetilde{\mathbf{K}}$ are worked out:

$$
\widetilde{\mathbf{K}} \mathbf{A}=\operatorname{MA} \mathbf{\Lambda} \text {, where } \mathbf{A}=\left[\alpha_{1} \alpha_{2} \ldots \alpha_{M}\right], \boldsymbol{\Lambda}=\operatorname{diag}\left\{\lambda_{1}, \lambda_{2}, \ldots, \lambda_{M}\right\}
$$

A is the orthogonal component space of D. For dimension reduction, $d(d<<N)$ principle components (that is, $\alpha$ corresponding to larger $\lambda$ ) are extracted to form feature space $\mathbf{F}$. For a given testing sample $y$, the feature $f$ can be calculated by (4).

$$
f=\mathbf{F}^{T} \mathcal{D}\left(\left[\Phi\left(x_{1}\right) \cdot \Phi(y) \Phi\left(x_{2}\right) \cdot \Phi(y) \cdots \Phi\left(x_{M}\right) \cdot \Phi(y)\right]^{T}\right)
$$

It should be noticed that $\mathbf{K}$ but not $\Phi$ is used directly in the algorithm, thus kernel projection is represented by the kernel matrix but not $\Phi$. In another words, nonlinear mapping is implemented by defining a relation measure (1) between all the samples. Three most popular classes of kernel functions are polynomial kernels, Gaussian kernels and sigmoid kernels. The choice of kernel is always done by experiment.

$$
K(x, y)=(x \cdot y)^{\text {degree }}
$$

Fractional power polynomial model [7] is employed in our system, where the power degree is set to 0.7 and feature dimension $d$ is set to 60 to maximize the performance to palmprint authentication. To lower the system complexity, finger feature extraction uses the same parameters as palmprint.

\subsection{Fusion Algorithm}

Given training samples of palmprint $\left\{p_{i}\right\}$ and finger samples $\left\{s_{i}\right\}(i=1, \ldots, M)$, corresponding new handmetric feature $\left\{h_{i}\right\}$ could be acquired using kernel method. First, kernel matrixes of palmprint and finger are:

$$
\left[\mathbf{K}_{p}\right]_{i j}=\left(p_{i} \cdot p_{j}\right)^{0.7},\left[\mathbf{K}_{s}\right]_{i j}=\left(s_{i} \cdot s_{j}\right)^{0.7}, i, j=1,2, \cdots, M
$$

For different biometrics, the same kernel is applied. Therefore, the kernel matrixes have general measurement. According to decision template method proposed by Kuncheva et al. [9], these matrixes could be fused reasonably. We define a fusion operator $\mathcal{B}$ to generalize different fusion rules. The four most popular rules in decision level fusion (sum, product, min and max) are adopted to evaluate the performance of our method. The new kernel matrix for handmetric $\mathbf{K}_{h}$ using different $\mathcal{B}$ could be:

$$
\begin{aligned}
& \mathcal{B}_{1}:\left[\mathbf{K}_{h-a v g}\right]_{i j}=\mathcal{D}\left(\left(\left[\mathbf{K}_{p}\right]_{i j}+\left[\mathbf{K}_{s}\right]_{i j}\right) / 2\right) ; \mathcal{B}_{2}:\left[\mathbf{K}_{h-p r d}\right]_{i j}=\mathcal{D}\left(\left[\mathbf{K}_{p}\right]_{i j} \cdot\left[\mathbf{K}_{s}\right]_{i j}\right) \\
& \mathcal{B}_{3}:\left[\mathbf{K}_{h-\min }\right]_{i j}=\mathcal{D}\left(\min \left(\left[\mathbf{K}_{p}\right]_{i j},\left[\mathbf{K}_{s}\right]_{i j}\right)\right) ; \mathcal{B}_{4}:\left[\mathbf{K}_{h-\max }\right]_{i j}=\mathcal{D}\left(\max \left(\left[\mathbf{K}_{p}\right]_{i j},\left[\mathbf{K}_{s}\right]_{i j}\right)\right)
\end{aligned}
$$


Note that operator $\mathcal{D}$ should act on the hold kernel matrix. We simplified the form (7) for better understanding.

The principle components of $\mathbf{K}_{h}$ span the handmetric feature space $\mathbf{F}$. The dimension of handmetric is set to 60, which is the same as palmprint and finger features.

After the training stage, handmetric feature of all the samples could be calculated. Suppose $p_{t}, s_{t}$ to be a pair of testing palmprint and finger samples, their kernel projections are:

$$
k_{p}=\left[\left(p_{1} \cdot p_{t}\right)^{0.7}\left(p_{2} \cdot p_{t}\right)^{0.7} \cdots\left(p_{M} \cdot p_{t}\right)^{0.7}\right]^{T}, \quad k_{s}=\left[\left(s_{1} \cdot s_{t}\right)^{0.7}\left(s_{2} \cdot s_{t}\right)^{0.7} \cdots\left(s_{M} \cdot s_{t}\right)^{0.7}\right]^{T}
$$

The relation between the testing handmetric to trained handmetric is:

$$
k_{h}=\mathcal{D}\left\{\mathcal{B}\left(k_{p}, k_{s}\right)\right\}
$$

And the handmetric $f_{h}$ would be:

$$
f_{h}=\mathbf{F}^{T} k_{h}
$$

By using (8)(9)(10), all the training and testing samples are projected in the handmetric space to extract handmetric features for classification.

\section{MAP Classifier for Verification}

The maximum a posteriori (MAP) classifier is designed for handmetric verification. In the training stage, every handmetric feature would minus all the other features to get the feature differences in the training set. If the difference is got form the same person, then it should be marked as "G (Genuine)" class. Otherwise, it is signed to "I (Impostor)" class. Classifier would build on the G and I classes in training samples.

In the testing stage, suppose a testing handmetric feature $f_{t}$ has the identity statement $Z$, according to [8], the difference $\Delta$ between $f_{t}$ and class center of $Z$ is treated as a sample input to the classifier, that is:

$$
\Delta=f_{t}-\overline{f_{Z}}, \quad \overline{f_{Z}}=\frac{1}{L}\left(\sum_{i=1}^{L} f_{i}\right), f_{i} \in Z
$$

The likelihood of $\Delta$ to $\mathrm{G}$ and I are:

$$
P(\Delta / G)=\frac{\exp \left\{-1 / 2\left(\Delta^{T} \Sigma_{G}^{-1} \Delta\right)\right\}}{(2 \pi)^{D / 2}\left|\Sigma_{G}\right|^{1 / 2}}, P(\Delta / I)=\frac{\exp \left\{-1 / 2\left(\Delta^{T} \Sigma_{I}^{-1} \Delta\right)\right\}}{(2 \pi)^{D / 2}\left|\Sigma_{I}\right|^{1 / 2}}
$$

Under the assumption that the prior is $P(G)=P(I)=1 / 2$ [14], we could get the posteriori probability $P(G / \Delta)$ :

$$
P(G / \Delta)=\frac{P(\Delta / G) P(G)}{P(\Delta / G) P(G)+P(\Delta / I) P(I)}=\frac{P(\Delta / G)}{P(\Delta / G)+P(\Delta / I)}
$$


Finally, comparing $P(G / \Delta)$ to similarity threshold $T$, we could draw the conclusion whether the identity statement is true.

\section{Experiments and Discuss}

A handmetric verification system is implemented based on our KPCA FLF method. A special device for hand image acquisition is designed. The device is made up of a CCD camera and a platform to fix the hand and illumination. 1,853 right hand images from 98 individuals are captured and stored in the database. For each person, up to $28 \mathrm{im}$ ages are captured in the period of 6 months for 4 times at most (the average is 2.7 times), 4 samples of each person are taken out to form a training set, while the remaining 1,481 images are taken as testing set. After preprocessing, palmprint database and finger database derived from hand image database. For each biometric (including palmprint, finger and handmetric) in the verification test, a total of $145,138(1481 \times 98)$ comparisons are performed for the testing images, in which $1,481(1481 \times 1)$ are genuine matching. The verification system is programmed using Matlab6.2 under Microsoft Windows XP environment, with Intel P4 2.6G CPU and 256MB memory.

\subsection{Comparison the Performance of Palmprint, Finger and Handmetric}

The aim of the first test is to verify the performance of handmetric. Whereas we proposed four fusion operators in 2.3 , here we choose $\mathcal{B}_{2}$ in (7) to demonstrate the

Table 1. Error rates for different biometrics

\begin{tabular}{ccccc}
\hline $3 / 4$ training samples & FAR $(\%)$ & FRR $(\%)$ & HTER $(\%)$ & EER $(\%)$ \\
\hline Palmprint & $0.51 / 0.45$ & $0.61 / 0.61$ & $0.56 / \mathbf{0 . 5 4}$ & $0.61 / \mathbf{0 . 5 5}$ \\
Finger & $0.70 / 0.72$ & $1.49 / 1.28$ & $1.10 / \mathbf{1 . 0 0}$ & $1.20 / \mathbf{1 . 0 7}$ \\
Handmetric & $0.23 / 0.29$ & $0.34 / 0.20$ & $0.48 / \mathbf{0 . 2 5}$ & $0.35 / \mathbf{0 . 2 8}$ \\
\hline
\end{tabular}
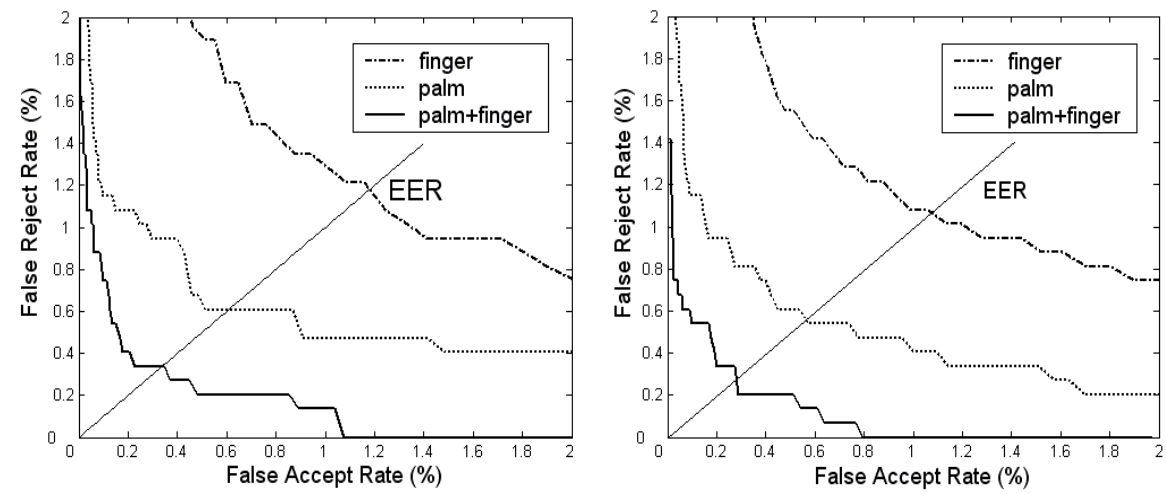

Fig. 2. ROCs of palmprint, finger and handmetric verification: (a) 3 training samples for each person, (b) 4 training samples for each person 
effectiveness of fusion algorithm. The test is carried out using 3 and 4 training samples for each person respectively (none of the biometrics works well using 2 samples through the test, the EERs (equal error rates) are higher than 10\%) to get a more generalized result.

Experimental results are given in Tab.1 and Fig.2. It is evident that feature extraction using KPCA and the MAP classifier are effective, most of the error rates are under $1 \%$. Furthermore, the improvement of handmetric is outstanding in both test, the EER of it reaches $0.28 \%$ using 4 training samples for each person, whereas palmprint is $0.55 \%$ and finger is $1.07 \%$ respectively in the same case. And, more training samples could make better result. As to handmetric, HTER turns to $0.25 \%$ from $0.48 \%$ when using one more sample.

\subsection{Comparison of Different Fusion Operators}

Different fusion operators affect the performance of handmetric greatly. Though the results in 4.1 show that 4 samples case would achieve better result, here we use only 3 samples to make the results of comparison more clearly.

As shown in Fig.3, the sum operator $\mathcal{B}_{1}$ performs the best in all the four operators, which is consist with most experimental results of decision level fusion [1][5][12]. Both of HTER (half total error rate) and EER reach $0.20 \%$ using only 3 samples

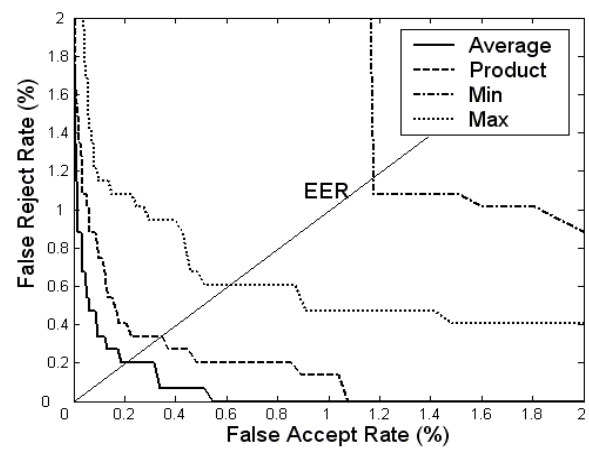

Fig. 3. ROCs of different fusion operators for each person, which shows the power of FLF method. However, the $\max$ operator $\mathcal{B}_{3}$ and min operator $\mathcal{B}_{4}$ couldn't work as well as $\mathcal{B}_{1}$ and $\mathcal{B}_{2} . \mathcal{B}_{3}$ could only maintain the accuracy of palmprint with little improvement, while $\mathcal{B}_{4}$ just gain a little than finger feature. Therefore, fusion cannot ensure the system performance absolutely, and proper fusion algorithm is needed to make it feasible.

Based on the test of 4.2 and 4.3 , we use operator $\mathcal{B}_{1}$ in 4 samples case to buildup the final handmetric verification system. The HTER and EER reach $0.11 \%$ and $0.07 \%$ respectively, which testifies that our FLF algorithm based on KPCA is effective, and handmetric is reliable and ready to application.

\section{Conclusion}

A novel FLF method based on KPCA is presented in this paper, and a handmetric verification system is set up based on it. Space incompatible and dimension curse problem of FLF are figured out by proposed method. The experimental results demonstrate the effectiveness of proposed method. The HTER and EER reach $0.11 \%$ and $0.07 \%$ respectively in the test database include 1,853 samples with 145,138 comparisons. 


\section{References}

1. Ross, A., Jain A. K.: Information Fusion in Biometrics. Pattern Recognition Letters, Vol. 24, (2003) 2115-2125

2. Lanckriet, G., Deng, M., Cristianini, N., Jordan, M. I.: Kernel-based Data Fusion and Its Application to Protein Function Prediction in Yeast. Proceedings of the Pacific Symposium on Biocomputing, (2004) 300-311

3. Li, Q., Qiu Z., Sun, D.: Personal Identification Using Knuckleprint, Sinobiometric04', Lecture Notes in Computer Science, Vol. 3338. Springer-Verlag, (2004) 680-689

4. Kumar, A., Wong, D., Shen, H. C., Jain, A. K.: Personal Verification using Palmprint and Hand Geometry Biometric. Proceedings of the fourth International Conference on audioand video-based biometric personal authentication, (2003) 668-678

5. Rabaric, S., Ribaric, D., Pavesic, N.: A Biometric Identification System Based on the Fusion of Hand and Palm Features. Proceedings of The Advent of Biometrics on the Internet, A Cost 275 Workshop. (2002)

6. Scholkopf, B., Smola, A., Muller, K. R.: Nonlinear Component Analysis as a Kernel Eigenvalue Problem. Neural Computation, Vol. 10, (1998) 1299-1319

7. Liu, C-j.: Gabor-based Kernel PCA with Fractional Power Polynomial Models for Face Recognition. IEEE Trans. PAMI, Vol. 26, (2004) 572-581

8. Moghaddam, B.: Principal Manifolds and Probabilistic Subspaces for Visual Recognition. IEEE Trans. PAMI, Vol. 24 (2002) 780-788

9. Kuncheva, L. I., Bezdek, J. C., Duin, R. P. W.: Decision Templates for Multiple Classifier Fusion: An Experimental Comparison. Pattern Recognition, Vol, 34 (2001) 299-314

10. 10.Zhang, D., Kong, W. K., You, J.: Online Palmprint Identification. IEEE Trans. PAMI, Vol 25 (2003) 1041-1050

11. Lu, G., Zhang, D., Wang, K.: Palmprint Recognition using Eigenpalms Features. Patter Recognition Letters, Vol. 24 (2003) 1463-1467

12. Jain, A. K., Ross, A.: Multibiometric Systems. Communication of the ACM, Special Issue on Multimodal Interfaces, Vol. 47 (2004) 34-40 